the previously reported PsA risk allele (HLA-B*27). Importantly, the study demonstrates that this additional information is efficiently captured using information theoretic criteria methods which capture correlations between markers.

Disclosure of Interest: None declared

DOI: 10.1136/annrheumdis-2018-eular.4430

\section{THU0007 GENETIC VARIANTS IN SLE SUSCEPTIBILITY LOCI, XKR6 AND GLT1D1, ARE ASSOCIATED WITH CHILDHOOD-ONSET SLE IN A KOREAN COHORT}

Y.B. Joo ${ }^{1}$, J. Lim² ${ }^{2}$, B.P. Tsao ${ }^{3}$, S.K. Nath ${ }^{4}$, K. Kim² ${ }^{2}$, S.-C. Bae ${ }^{5}{ }^{1}$ St. Vincent's hospital, The catholic university of Korea, Suwon; ${ }^{2}$ Kyung Hee University, Seoul, Korea, Republic of Ireland; ${ }^{3}$ Medical University of South Carolina, Charleston, South Carolina; ${ }^{4}$ Oklahoma Medical Research Foundation, Oklahoma, USA; ${ }^{5}$ Hanyang University Hospital for Rheumatic Diseases, Seoul, Korea, Republic of Ireland

Background: Systemic lupus erythematosus (SLE) is a polygenic autoimmune disease that occurs in all ages. It has been well documented that younger age of SLE onset is associated with more severe clinical manifestations and worse outcomes. However, impact of genetic variants on age of SLE onset was not fully understood.

Objectives: We investigated a cumulative effect of reported SLE-risk variants on childhood-onset SLE and searched for new risk loci of childhood-onset SLE using a genome-wide SNP analysis.

Methods: We analysed 96 Korean childhood-onset ( $<16$ years old) and 685 adult-onset SLE ( $>16$ years old) who were previously genotyped by both Immunochip and genome-wide SNP arrays. Individual genetic risk scores (GRS) from well-validated SLE susceptibility loci (45 Asian confirmed non-HLA loci and an HLA-DRB1 amino acid haplotype model) were calculated and tested for their association with childhood-onset SLE. Association of each genetic variant with childhood-onset SLE was analysed using a multivariable logistic regression adjusting for population substructure.

Results: Mean age of SLE onset was $12.5 \pm 2.5$ years in childhood-onset SLE and 29.0 \pm 9.4 in adult-onset SLE. GRS from SLE susceptibility loci was significantly higher in childhood-onset SLE than adult-onset SLE $(p=0.001)$. Two SNPs, rs7460469 in XKR6 and rs7300146 in GLT1D1, showed the most significant associations with childhood-onset SLE $(p=1.26 \times 10-8, O R=0.18$, and $\mathrm{p}=1.49 \times 10-8, \mathrm{OR}=0.35$, respectively). Especially, rs7300146 in GLT1D1 was the cis expression quantitative trait locus (eQTL) for SLC15A4, which has been known an SLE susceptibility gene in a Chinese population. The model consisting of SLE GRS and the two newly identified loci to predict childhoodonset SLE attained an area under curve (AUC) of 0.71 in a receiver operating characteristics (ROC) curve.
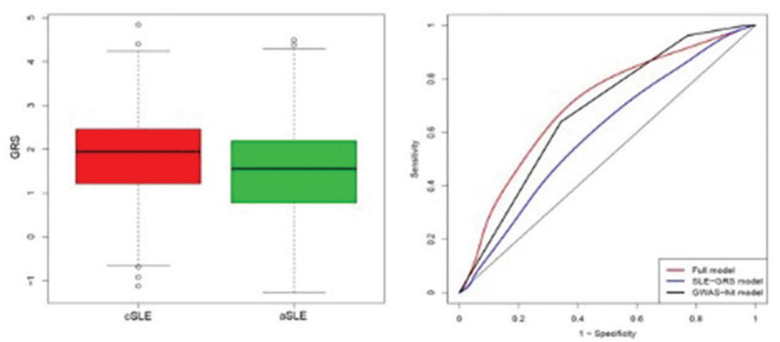

Abstract THU0007 - Figure 1. A) association of genetic risk score else. cSLE; childhoodonset, aSLE; adult-onset SLE, GRS; genetic risk score and B) ROC curve for SLE-GRS, GWAS-hitand full model. ROC; receiver opertating characteristics, SLE; Systemic lupus erythematosus, GWAS; genome-wide association study

Conclusions: Childhood-onset SLE is associated with a high cumulative SLErisk effect and two novel SNPs rs7460469 and rs7300146, providing the first predictive model for childhood-onset SLE in Koreans.

\section{REFERENCES:}

[1] Early disease onset is predicted by a higher genetic risk for lupus and is associated with a more severe phenotype in lupus patients. Ann Rheum Dis. 2011;70(1):151-156.
[2] Risk Alleles for Systemic Lupus Erythematosus in a Large Case-Control Collection and Associations with Clinical Subphenotypes. PLoS Genet. 2011 Feb;7(2):e1001311.

[3] High-density genotyping of immune-related loci identifies new SLE risk variants in individuals with Asian ancestry. Nat Genet. 2016;48:323-330.

[4] The HLA-DRß1 amino acid positions 11-13-26 explain the majority of SLE-MHC associations. Nat Commun, 2014:5:5902.

Disclosure of Interest: None declared

DOI: 10.1136/annrheumdis-2018-eular.4995

\section{THU0008 LINKING GENETICS TO T-CELL PHENOTYPE IN JIA: RATIONAL FOR IL-2 THERAPY}

A. Yarwood ${ }^{1,2}$, A. Hinks ${ }^{1}$, S. Smith ${ }^{1}$, J. Bowes ${ }^{1}$, S. Eyre ${ }^{1}$, W. Thomson ${ }^{1,2}$. ${ }^{1}$ Arthritis Research UK, Centre for Genetics and Genomics; ${ }^{2}$ NIHR Manchester Musculoskeletal Biomedical Research Centre, Manchester University NHS Foundation Trust, The University of Manchester, Manchester, UK

Background: Several genetic regions associated with susceptibility to juvenile idiopathic arthritis (JIA), harbour genes involved in the interleukin-2 (IL-2) response which is pivotal in the function of regulatory $T$ cells (T-regs) and their ability to supress effector T cells. Genetically JIA is similar to type 1 diabetes (T1D) which also demonstrates an enrichment of genes related to IL-2 regulation and response. Early clinical trial data has indicated recombinant IL-2 to be suc cessful in enhancing T-regs in T1D. I hypothesise that IL-2 regulation and response is critical to the development of JIA and therefore IL-2 therapy represents an exciting and viable therapeutic option for JIA.

Objectives: We aim to identify a subset of JIA patients who carry a high burden of genetic risk variants in genes related to IL-2 regulation and response, who could then be targeted for IL-2 therapy. Secondly we will link genetics to cellular phenotypes using CyTOF to identify a subset of cells that are most perturbed in JIA and determine the effects of IL-2 on these cellular subsets.

Methods: A weighted genetic risk score (wGRS) was generated using $9 \mathrm{JIA}$ susceptibility SNPS considered to be within or near to genes involved in interleukin (IL-2) regulation and response. ${ }^{1}$ The IL-2 wGRS was tested in an independent set of UK cases (1435) and controls (5181). The risk of developing JIA was assessed by subtype, using logistic regression. A CyTOF panel containing 33 antibodies targeting markers of $\mathrm{T}$ cells and T-regulatory cells was developed and tested in CD3 $+\mathrm{T}$ cells from two healthy individuals after 12 hour stimulation (anti CD3/CD28 beads plus recombinant IL-2). Cells were stained for all antibodies, iridium and cisplatin and analysed on the CyTOF Helios. Data will be analysed with traditional biaxial gating as well commercially available packages such as cytofkit.

Results: The IL-2 wGRS demonstrated an increased percentage of individuals in the high risk group in the extended oligoarthritis, RF negative and RF positive polyarthritis subtypes suggesting a higher burden of IL-2 related loci. The odds of developing JIA for those in the highest risk group (quintile 5) compared to all others was increased in these subtypes (OR 2 95\% Cl 1.45-2.76, OR 2.39 $95 \% \mathrm{Cl} 1.87-3.04$, OR $2.1495 \% \mathrm{Cl} 1.49-3.09$, respectively). Comparing this to a wGRS generated from JIA susceptibility loci excluding IL-2 related genes shows that this enrichment is specific to the IL-2 wGRS. Biaxial gating of CyTOF data showed increases in activation markers after stimulation (CD25, CD69, CD38 and HLA-DR, decrease CCR7). We demonstrated that our panel can successfully identify traditional CD4 $+\mathrm{T}$ cell subsets showing differences between stimulated and unstimulated cells and between individuals.

Conclusions: Our analysis has shown that patients with oligoarthritis and polyarthritis have an increased burden of JIA susceptibility variants in genes related to $\mathrm{IL}-2$ regulation and response suggesting these individuals may benefit from IL2 therapy. Using the CyTOF panel we can now analyse individuals with high and low GRS allowing us to identify cellular subsets which may be altered by these genetic variants.

\section{REFERENCE:}

[1] Hinks A, Cobb J, Marion MC, et al. Dense genotyping of immune-related disease regions identifies 14 new susceptibility loci for juvenile idiopathic arthritis. Nat Genet 2013;45(6):664-69.

Disclosure of Interest: None declared DOI: 10.1136/annrheumdis-2018-eular.6868 